\title{
ANALYZING THE PERFORMANCE OF WIRELESS LOCAL AREA NETWORKS WITH AN IMPROVED COLLISION AVOIDANCE MECHANISM
}

\author{
Pedro Braconnot Velloso, Miguel Elias Mitre Campista, Daniel de Oliveira Cunha, Luís Henrique \\ Maciel Kosmalski Costa, and Otto Carlos Muniz Bandeira Duarte
}

\begin{abstract}
In this paper we present and analyze a collision avoidance mechanism to increase the throughput and decrease the delay in wireless local area networks. The analysis considers the IEEE 802.11 Standard parameters employed in the Distributed Coordination Function used in the Ad Hoc mode operation. The mechanism is based on the deferral counter (DC) algorithm proposed in HomePlug Standard. The purpose of the Deferral Counter is to increase the contention window faster than the regular backoff function from IEEE 802.11 to prevent collisions. Different from IEEE 802.11, the mechanism increments the contention window of the deferring stations to minimize collisions. We implement this mechanism in ns-2 simulator in order to compare it with IEEE 802.11. We propose three different functions to use in the Deferral Counter mechanism and evaluate their performances considering network throughput, packet delay, and the percentage of overlapping transmissions. Results show that the proposed mechanism performs better when there is more than four active stations no matter the packet size. Furthermore, we also show that the DC function that achieves the best network throughput depends on the packet size and the number of active nodes.
\end{abstract}

Keywords: Medium Access Control (MAC), IEEE 802.11 networks, network capacity.

Resumo - Este trabalho tem como principal objetivo apresentar e analisar um novo mecanismo para aumentar a vaz ao e minimizar o atraso em redes locais sem fio. A an álise considera os parametros do padra ao IEEE 802.11 empregados na Func, ao de Coordenac, ao Distribu'ida utilizada na operac, ao em modo Ad Hoc. Este mecanismo baseia-se no algoritmo de contador de adiamento (DC) proposto pelo padrao de redes domicicilares HomePlug. $\mathrm{O}$ objetivo do DC 'e aumentar mais rapidamente, que a func, ao de backoff do IEEE 802.11, a janela de contenc, ao para evitar colis oes. Diferentemente do IEEE 802.11, o mecanismo incrementa a janela de contenção de estações em backoff para minimizar as coli $\sim$ oes. Este me-

\footnotetext{
This work was funded by FAPERJ, CNPq, CAPES, FINEP, and RNP. Pedro Braconnot Velloso ( $\mathrm{Ph}$. D student and CNPq-Brazil Scholar) is with the Laboratoire LIP6 - Universit'e Pierre et Marie Currie, 8, rue du Capitaine Scott, 75015, Paris, France. Miguel Elias Mitre Campista (M. Sc. Student and CNPq-Brazil Scholar), Daniel de Oliveira Cunha (Ph. D. student and CNPq- Brazil scholar), Lu'is Henrique Maciel Kosmalski Costa, and Otto Carlos Muniz Bandeira Duarte are with GTA/COPPE/POLI Universidade Federal do Rio de Janeiro, P.O. Box 68504, 21945 970, Rio de Janeiro, RJ, Brazil.
}

canismo foi implementado no ns-2 a fim de compar'a-lo com o mecanismo padr ao do IEEE 802.11. Sॅao proposta tr ${ }^{\wedge}$ es func, oes diferentes para serem usadas com o mecanismo de contador de adiamento com o objetivo de avaliar qual oferece o melhor resultado. Os parametros utilizados na an'alise de desempenho foram a vaz ao agregada, o atraso e a percentagem de transmiss oes sobrepostas. Os resultados mostram que o mecanismo proposto obteve um melhor desempenho para uma rede com mais de quatro n'os transmissores independentemente do tamanho do pacote. Verifica-se ainda que a func, ao de incremento do contador de adiamento que atinge melhor resultado para a capacidade da rede depende do tamanho dos pacotes e do n'umero de n'os transmitindo.

Palavras-chave: Controle de acesso ao meio, Redes IEEE 802.11, capacidade da rede

\section{INTRODUCTION}

Wireless communication is becoming more popular mainly due to its high flexibility and low installation cost. Nevertheless, the wireless medium imposes singular impairments to device designers. These impairments include low bandwidth, high bit error rate, and significant variations of the physical medium characteristics. In order to cope with these singularities, the design of efficient Medium Access Control (MAC) protocols for wireless networks is of utmost importance. The high attenuation compelled by the medium to transmitted signals makes it difficult to the emitter to detect collisions. As a consequence, the well known Carrier Sense Multiple Access with Collision Detection (CSMA/CD) [IEEE, 2002] mechanism used in the Ethernet MAC protocol is unsuitable to wireless networks. Therefore, the conventional solution for wireless networks is to avoid, as much as possible, the occurrence of collisions. In infrastructured wireless networks there is a central entity, typically an Access Point. This central entity can be exploited to support pooling access schemes that wipe out collisions. Ad hoc networks protocols, on the other hand, can not count on such mediators. Hence, ad hoc protocols are distributed and, typically, contention-based. The IEEE 802.11 is the most popular wireless local area network (WLAN) technology. The fundamental medium access method of the IEEE 802.11 is the Distributed Coordination Function [IEEE, 1999], also known as CSMA/CA (Carrier Sense Multiple Access with Collision Avoidance). The CSMA/CA protocol has two distinct features to minimize collisions: sensing the medium for a fixed amount of time prior to the transmission and a random backoff mechanism. The backoff mechanism sets a timer, which is composed by 
a random number uniformly distributed between zero and a contention window size $([0 ; C W])$ times a slot time. When the backoff timer goes off the station transmits its frame. Whenever a transmission fails, the current contention window size is increased by $2 £$ CWcurrent +1 . This increase happens up to a maximum threshold ( $C W \max$ ). The contention window of deferring stations, or stations not directly involved in the collision, do not change. Although the random backoff decreases the collision probability, it can also degrade the network efficiency by adding idle slots. Clearly, there is a tradeoff between the gain with the collision reduction and the wasted time with idle slots. There are different techniques aiming at increasing the medium access efficiency of WLANs. A first approach deals with the problem of how and when to increase the contention window. An important issue addressing this problem is the increasing of the contention window of the deferring stations. Other possibilities concern how the contention window must be decreased after a successful transmission and how to reduce the impact of idle slots. In this paper, we analyze a mechanism to increase the contention window of the deferring stations based on the number of transmissions sensed by the station during its backoff. The number of transmissions required for the augmentation of the contention window is dictated by a counter, denominated Deferral Counter (DC). The value of this counter depends on the backoff stage, which is related to the number of times the backoff function is called. Our work proposes and analyzes three possibilities for the evolution of the DC as a function of the backoff stage: a constant DC, a linear DC, and an exponential DC. The effects of the different approaches are evaluated based on the throughput and on the probability of collision occurrence. Our work is organized as follows. In Section 2 we present the related work. Section 3 gives a brief overview of the IEEE 802.11 MAC protocol. Section 4 introduces our proposed scheme and the deferral counter (DC) mechanism. We also show three possible functions for the deferral counter. Section 5 shows the algorithm applied and its simple implementation. Section 5 presents the efficiency of the mechanism through our simulation results. Finally, we conclude the paper in Section 6.

\section{RELATED WORK}

Several works derive analytical expressions for the network capacity of 802.11 networks [Li et al., 2001, Villela and Duarte, 2004, Tay and Chua, 2001]. These works are based on the average value of a variable, instead of a stochastic analysis. None of these works addressed the problem of fast increase of the contention window. Tay and Chua, also develop a mathematical model which allows to evaluate the best CWmin and CWmax, but not the backoff function.[Kuo and Kuo, 2003] propose a simple backoff scheme to DCF and an analytical model based on Markov chains toanalyze the throughput and delay performance of the new scheme. The analysis shows that the proposed algorithm can improve network throughput and reduce access delay. Although, the proposed scheme performs better than the IEEE 802.11 , they do not analyse the results for different backoff functions and their effects on different scenarios. [L.Bononi et al., 1998] propose a distributedcontentioncontrol mechanism to avoid collision and improve network capacity, by decreasing the mean access delay. The key idea is to distribute transmission attempts over a variable sizedtime window the most uniform as possible. The medium access algorithm is based on an estimation of the contention level, which is obtained by the slot utilization. Each station counts the number of busy slots during its own backoff interval. This value is divided by the backoff interval to obtain the slot utilization. This result is used to calculate a medium access probability that also depends on the number of unsuccessful attempts. They show that the proposed mechanism can improve network efficiency and it introduces no overhead in low load cases. Nevertheless, the contention window is not optimized and the algorithm might introduce a lot of idle slots. [Kwon et al., 2003] propose a mechanism that improves network throughput and fairness. In order to enhance the network throughput, the proposed scheme is based on two assumptions: fast collision resolution and reduction of idle slots. The fast collision resolution is achieved by changing the backoff window size for the deferring stations whenever they sense a busy slot. In addition, they use a larger CWmax, when compared to the one of 802.11 , to reduce the collision probability. Idle slots is minimized by setting a much smaller $C W m i n$ and decreasing backoff timers exponentially when a prefixed number of consecutive idle slots are detected. The paper also presents an extension to this mechanism to provide medium access fairness. This paper focus on the problem of fast increase of the contention window. We investigate the functions that can be used to increase the contention window for the deferring stations. The simulations take into account the number of active stations and the packet size. The effects of the different approaches are evaluated based on the throughput and on the probability of collision occurrence.

\section{THE IEEE 802.11 MAC PROTOCOL}

The IEEE 802.11 standard defines the Medium Access Control (MAC) and Physical Layer (PHY) specifications. In Distributed Coordination Function (DCF) mode, whenever a station wants to transmit a data frame, it must wait the medium to remain idle for DIFS (Distributed Interframe Space) which lasts for $50^{I} \mathrm{~s}$. After DIFS, the station transmits its data frame as illustrated in Figure 1. Due to medium attenuation, which make collision detection not feasible, the transmitter waits an acknowledgment (ACK) from the receiver. The receiver transmits an ACK whenever it receives correctly a data frame. The ACK frame is transmitted after the end of the data frame reception plus a time interval SIFS (Short Interframe pace), which lasts for $10^{1} \mathrm{~s}$. As SIFS is shorter than DIFS, the ACK transmission is guaranteed before any other data transmission. To avoid collisions, after the first data frame, all the transmitted data frame waits for DIFS or EIFS (Extended Interframe Space) plus a random time (backoff). DIFS is used if the last transmission detected on the medium was received correctly, and EIFS, if it was not. The stations defer for EIFS, which is larger then DIFS, whenever they were not able to determine the situation of 
the current transmission progress. The reception of an erroneous frame makes the stations unaware of what frame was received. Thus, they must wait a sufficient time interval necessary for a possible current transmission ends. The EIFS interval follows the reception of the erroneous frame supposing it was a data frame. The EIFS avoids collisions between data and ACK frames. It lasts for a SIFS plus a period corresponding to an ACK frame transmission plus a DIFS. The backoff mechanism is activated after DIFS or EIFS. The backoff function chooses a random number uniformly distributed between zero and the contention window size $[0 ; C W]$. This number is used as a backoff counter and its value times a slot time $\left(20^{\prime} \mathrm{s}\right)$ composes a backoff timer. A slot of $20^{I} \mathrm{~s}$ is a suitable interval of time to guarantee the frame detection of an upcoming transmission by all the stations of the network. The stations decrement by one their backoff counter if the medium remains idle for a slot time. They keep decrementing until the backoff timer goes off and the transmission occurs. Nevertheless, if the medium is occupied while a station is waiting for backoff, the station must pause its backoff counter and wait the next transmission attempt to resume it. The backoff function is called if the transmitter does not receive an ACK frame from the receiver. The backoff function takes care of the increase of contention window size. This size depends on the number of times the backoff function is called during the transmission of one specific frame. The backoff function is called (Backoff Function Calls - BFC) every time the corresponding ACK frame is not received and, as a consequence, a collision is supposed to have occurred. Therefore, the contention window increase avoids collisions by decreasing their occurrence probability. In the first attempt to access the medium, the station chooses a value to its backoff counter between zero and the minimumcontention-window size $[0: C W m i n](C W m i n=31)$. The increase in the contention-window size follows the expression $2 £ C W$ current +1 , where $C W$ currentis the current $\mathrm{CW}$ size used in the transmission. This increase continues up to a maximum threshold ( $C W \max =1023$ ). If the backoff function is called when $\mathrm{CW}$ is already in its maximum value ( $C W$ max), the $C W$ size is not changed. $C W$ returns to its initial value whenever a successful transmission occurs. The values of the contention window $(\mathrm{CW})$ are shown in Table 1 .

Table 1. Contention window (CW)

\begin{tabular}{|c||c|c|c|c|c|c|}
\hline BFC & 0 & 1 & 2 & 3 & 4 & 5 \\
\hline$C W$ & 31 & 63 & 127 & 255 & 511 & 1023 \\
\hline
\end{tabular}

The DCF implements a virtual allocation vector called

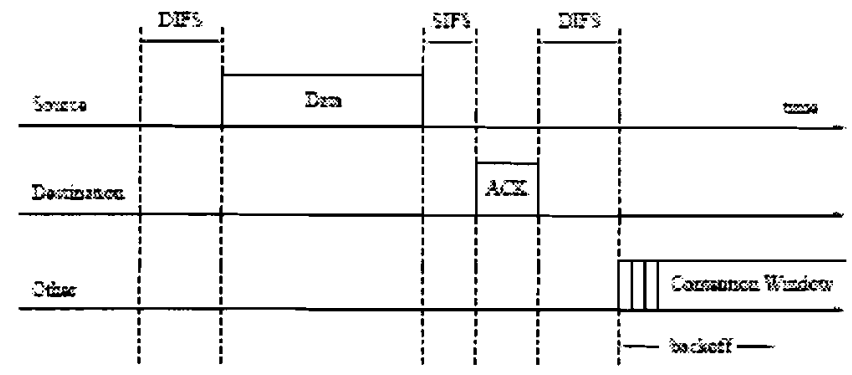

Figure 1. Distributed Coordination Function (DCF) basic scheme.

NAV (Network Allocation Vector). When a station is listening to the medium, it is aware about the duration of the current transmission through the available information on the ongoing frames. Hence, stations know for how long the medium will be busy and, as a consequence, for how long they will not be able to transmit. This scheme avoids collisions. The RTS/CTS (request-to-send/clear-to-send) mechanism is used to carry the transmission duration, besides avoiding the hidden node problem. Every RTS and CTS frames are used to update the NAVs from the stations that are contending for the medium. When the RTS/CTS mechanism is employed, the transmitter sends the RTS after DIFS. After receiving a RTS frame, the receiver transmits the CTS after SIFS. When the transmitter receives the CTS, the RTS/CTS exchange is complete. Then, it transmits a data frame after SIFS as shown in Figure 2. Following the data transmission, the transmitter waits for an ACK as in the basic scheme. Every station must be able to hear the RTS or CTS frames since they are inside the transmission range of the RTS/CTS transmitter. Therefore, the RTS/CTS frames must be transmitted at the basic rate, usually at $1 \mathrm{Mbps}$

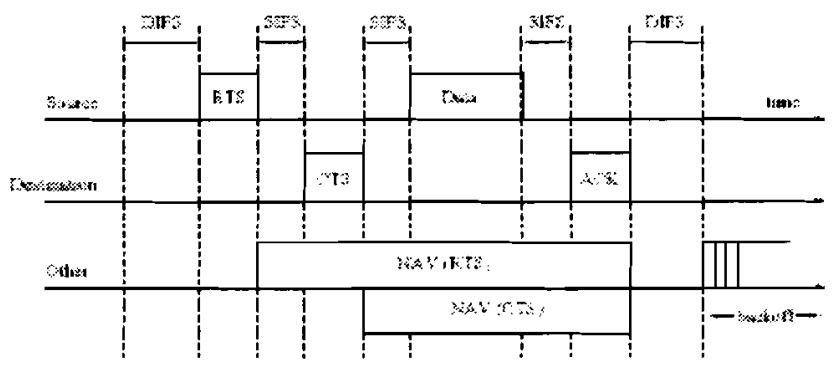

Figure 2. Distributed Coordination Function (DCF) with RTS/CTS.

\section{USING DC OVER IEEE 802.11}

The maximum performance of the CSMA/CA is achieved when one frame is transmitted after the other without collisions and idle slots. In other words, to increase the performance, collisions must be avoided at the same time that the backoff must be minimized. Our proposition is an improvement in the backoff mechanism which allows better throughput and lower delay. This is due to the employment of a mechanism that decreases the collision rate. This mechanism, called deferral counter (DC), was introduced by the Home-Plug standard ([Lee et al., ]) used in PowerLine Communications. The HomePlug defines the MAC protocol used in high speed data transmissions in HomeNetworks through the electrical wiring. The deferral counter was conceived to work along with the backoff mechanism to avoid collisions. The DC is decremented whenever a station senses the medium being captured by another station. When the DC reaches zero, the node assumes that there is a considerable number of stations trying to transmit, which increases the collision probability. Thus, a station must call the backoff function when it senses that the medium was captured again and increment its deferral counter, as seen in Figure 3. 
1 transmit 9

Wait to transmi: âsme:

if medum is vale for DIFS then star, backot:

if broliof is started and the medurn getc bus then Verif $\mathrm{DC}$.

if $\mathrm{DC}=0$ then

stop_backofi:

macrensent $D C$

iscrenent_Cs:

retrin:

eise

decrement $D C$.

pause_oncioft:

reture;

else if bacloeff tmer $==0$ and toe wediun $==$ idle thes

transmit the îne and wais the acknowledgment:

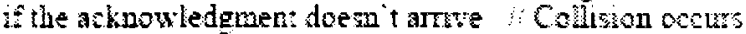
increment $D C$ :

iscrement_CH:

retur:

else

re:ur:

Figure 3. The algorithm of the improved collision avoidance mechanism.

The values of the contention window and the corresponding values of the deferral counter used in HomePlug are in Table 2. The HomePlug uses different channel access priorities (CAP), ranging from $\mathrm{CA} 3$ to $\mathrm{CA} 0$, where $\mathrm{CA} 3$ is the highest priority. This classes are assigned to provide quality of service.

Table 2. Contention window $(\mathrm{CW})$ and deferral counter (DC) values defined in the HomePlug Standard.

\begin{tabular}{|c||c|c|c|c|}
\cline { 2 - 5 } \multicolumn{1}{c|}{} & \multicolumn{2}{c|}{ CAP: CA3, CA2 } & \multicolumn{2}{c|}{ CAF: CA1, CA0 } \\
\hline BEC & CW & DK & CW & IX: \\
\hline \hline 0 & 7 & 0 & 7 & 0 \\
\hline 1 & 15 & 1 & 15 & 1 \\
\hline 2 & 15 & 3 & 31 & 3 \\
\hline$\therefore 2$ & 31 & 15 & 63 & 15 \\
\hline
\end{tabular}

Table 3 shows an example of the evolution of $\mathrm{CW}$ and DC. At instant 0 , suppose that a node attempts, for the first time, to access the medium. Nevertheless, some other node with the same priority has captured the medium first. As a consequence, the node that was unable to transmit, calls the backoff function and also increases its DC to the next value, equal to 1 . At instant 2 , the node transmits, but a collision occurs compelling it to increase its contention window and its DC. At instants 3 and 4 , the node can access the medium and transmit its frames, setting its $\mathrm{CW}$ size and DC at the minimum values. Starting at instant 5 , the node is unable to transmit because the medium is always busy due to other transmissions. The DC value is decreased up to reaching zero. Occurring another transmission, the node calls the backoff function again and once more increases its DC to the next value.

Table 3. Example of backoff and deferral counter (DC) evolution.

\begin{tabular}{|c||c|c|}
\hline Inatant & Backoff & DC \\
\hline \hline 0 & 7 & 0 \\
\hline 1 & 15 & 1 \\
\hline 2 & 31 & 3 \\
\hline 3 & 7 & 0 \\
\hline 4 & 7 & 0 \\
\hline 5 & 15 & 1 \\
\hline 6 & 15 & 0 \\
\hline 7 & 31 & 3 \\
\hline 8 & 31 & 2 \\
\hline 9 & 31 & 1 \\
\hline
\end{tabular}

This paper implements and analyzes the adoption of the deferral counter by the IEEE 802.11. In order to do that, we have proposed three possibilities for the deferral counter values comparing them with the case without $\mathrm{DC}$. The first proposition keeps the value of the DC constant; the second, increases the DC values obeying a linear function of type 4 $£ n+3$; and the third, increases the DC values obeying an exponential function of type $2(n+2) ; 1$. The minimum value of the deferral counter was chosen as three, because the IEEE 802.11 has a $C W_{\min }$ value higher than Home-Plug. Thus, the collision probability is lower and the deferral counter may be less efficient if its initial value is zero. This would represent a high overhead, specially when a few number of nodes are trying to transmit. Moreover, when the $\mathrm{CW}$ reaches its maximum value $\left(C W_{\max }\right)$, the $\mathrm{DC}$ value stops being increased also. Table 4 shows the evolution of the $\mathrm{DC}$ value as the $\mathrm{CW}$ increases for the three proposed functions.

Table 4. Bactoef and DC exolution of the proposed schenes.

\begin{tabular}{|c||c|c|c|}
\cline { 2 - 4 } \multicolumn{1}{c|}{} & \multicolumn{3}{c|}{ Deferral counter (DC) } \\
\hline \hline 31 & 3 & 3 & 3 \\
\hline 63 & 3 & 7 & 7 \\
\hline 127 & 3 & 11 & 15 \\
\hline 255 & 3 & 15 & 31 \\
\hline 511 & 3 & 19 & 63 \\
\hline 1023 & 3 & 23 & 127 \\
\hline
\end{tabular}




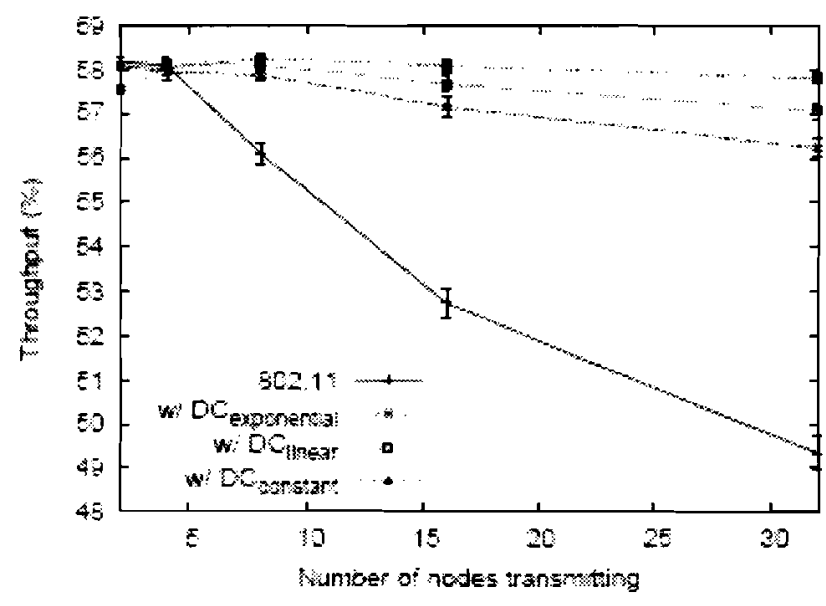

(a) Throglipu for 1500 bytes packes.

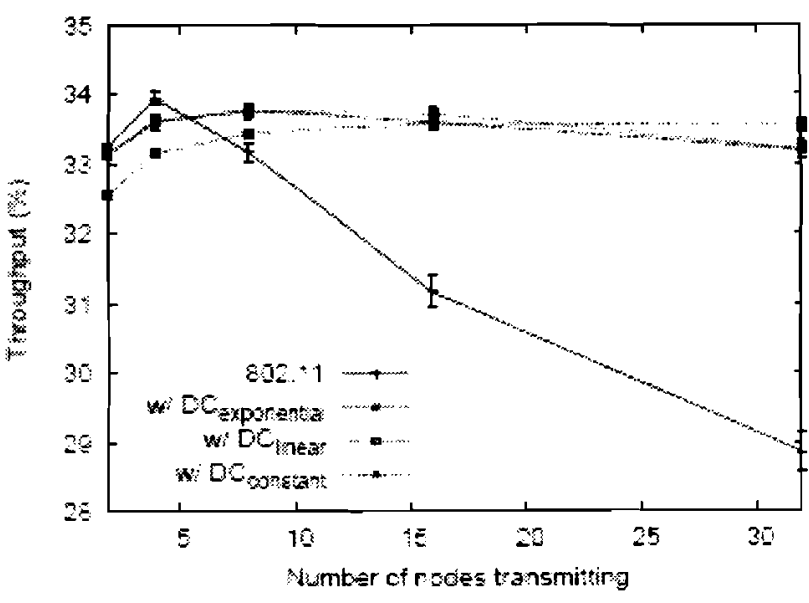

(b) Turugisput for 31 ? bre: pades:

Figure 4. Comparison of the throughput with and without the analyzed mechanism.

\section{RESULTS}

To implement the DC mechanism, we modify the IEEE 802.11 module available in the Network Simulator 2 (ns-2) [Fall and Varadhan, 2002]. We altered the backoff mechanism in order to achieve the behavior shown in Figure3. Whenever a station wants to transmit a frame, the station must wait for DIFS and for a backoff time successively. When waiting for backoff, if a station senses the medium getting busy, the stations must verify its DC. If its DC is zero, the station must stop its backoff, increment $\mathrm{DC}$ and $\mathrm{CW}$, and wait the medium to get idle again. If the DC is not zero, the station must decrement it and proceed as the usual backoff mechanism employed in the IEEE 802.11, namely pausing the backoff timer and waiting the medium to get idle again to resume it. A transmission occurs whenever the backoff timer reaches zero. After transmitting a data frame, the station waits for an acknowledgment. If the transmitter does not receive an acknowledgment, it must increment its $\mathrm{DC}$ and $\mathrm{CW}$ because a collision must have happened. The scenario consists of a single hop network with 32 nodes randomly placed in an $150 \mathrm{~m} \times 150 \mathrm{~m}$ area. Thus, every station can directly communicate with each other. Active stations, namely, the ones that is transmitting, is modeled by a CBR source and we assume that a packet is always available for transmission. We ran a set of simulations varying the number of active stations. The main goal is to assess the behavior of the network capacity according to the backoff function and the number of active stations. The results consider $95 \%$ of confidence intervals for the mean that are represented in the figures by vertical bars. Figure 4(a) shows the network throughput for packets of 1500 bytes. The first thing to notice is that all three DC functions perform better than without $D C$, except for less than 5active stations. In such case, the throughout behaves almost the same when using the regular backoff function (without DC), the linear DC function, and the exponential DC function. The purpose of DC is to augment network capacity by reducing the number of collisions. When the total active nodes is less than $\mathbf{5}$, there is a low collision probability. Therefore, while the collision probability is negligible, we can increase the network throughput by increasing the number of active stations. Because we are decreasing the amount of idle slots related to backoff. Actually, the average backoff time is divided by the number of stations. This behavior is presented in Figure $4(\mathrm{~b})$, where the curve related to the regular backoff function shows a small elevation and then after 4 stations it goes down. In this particular case, when collision probability is negligible, the regular backoff function performs better than the other ones because, in fact, there is no need to avoid collisions. As the number of active nodes increases, the efficiency of the regular backoff drops fast while the ones that use DC perform much better. Figure 4 (b) shows that the linear and exponential backoff functions perform better than the constant function until 16 active stations, which means we do not need to increase the contention window as fast as the constant function does for a small number of stations. It's worth to mention that the effect of a collision on the network throughput depends on the size of the packet. The larger is the packet the worse is the effect. This is the reason in Figure 4(a) there is no improvement in network capacity when there is less than 5 stations. Although a small number of collisions occurs, the decreasing in average backoff time is not enough to overcome the cost of the collisions. Figures 5(a) and 5(b) present the impact of the number of active stations on packet delay. It is clear, in both figures, that the deferral counter mechanism can decrease the end-toend delay. However, the curves did not reflect the network improvement achieved by DC mechanism, as seen in Figures 4(a) and 4(b). End-to-end delay comprises not only the backoff delay, but also the queuing delay. When the network operates in saturation the queuing delay might accumulate several backoff delays and reach a considerable value. This problem can make the backoff delay almost negligeable for a small number of active nodes. Figures $6(\mathrm{a})$ and $6(\mathrm{~b})$ illustrate the percentage 


\section{P.B. Velloso, M.E.M. Campista, D.O. Cunha, L.H.M.K. Costa, and O.C.M.B. Duarte Analyzing the Performance of Wireless LANs with an Improved Collision Avoidance Mechanism}

of overlapping transmissions. We can observe that the size of the packet do not affect the percentage of overlapping transmissions. The collision probability is independent from the packet size, thus for smaller packets there will be more overlapping trans-

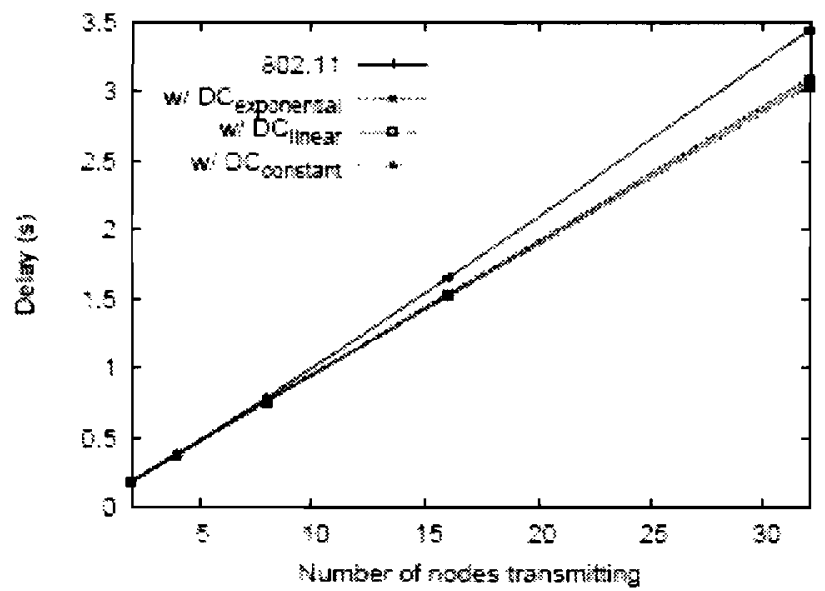

(a) Deisy far 1500 byter packets.

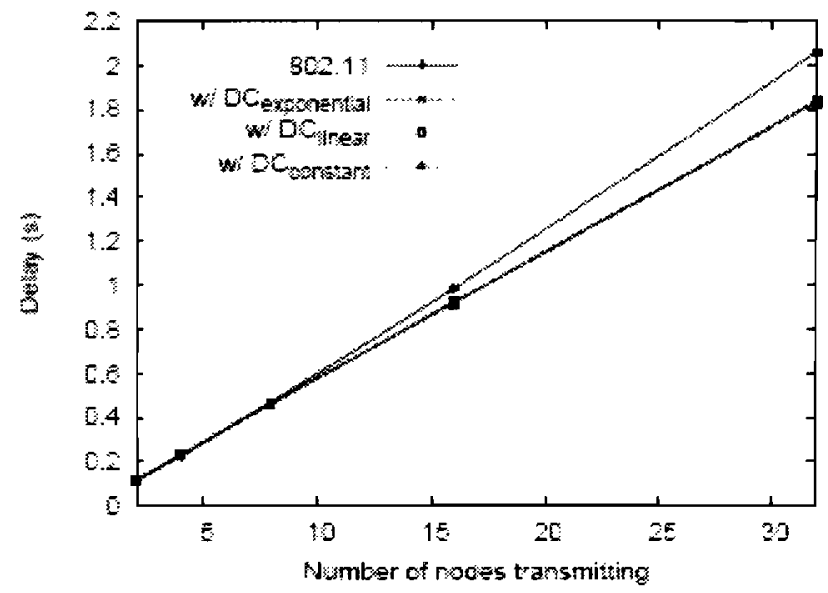

b) Delay for 522 byte packet:

Figure 5. Comparison of the delay with and without the analyzed mechanism

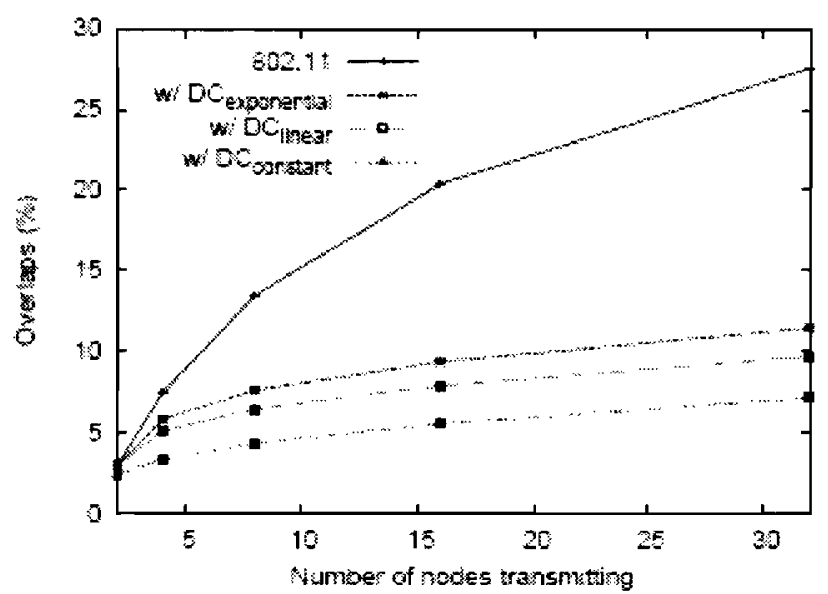

(a) Oreriapping for 500 bites paches:

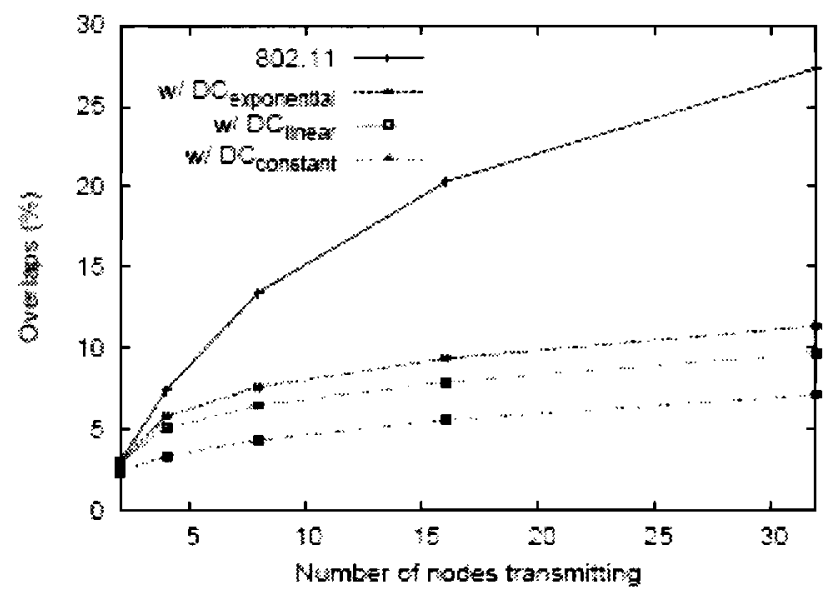

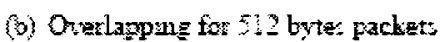

Figure 6. Comparison of the overlapping with and without the analyzed

missions. However, in the same amount o time, the proportion between the number of successful sent packets and overlapping transmission for small and large packets are the same. Figure $6(\mathrm{~b})$ shows that the percentage of overlapping transmissions for the regular backoff functions is higher than the others. An expected result is that the curve disposition is ordered by the ability to enlarge the contention window. The faster it does the lower is the percentage of overlapping transmissions. The interesting observations is that just increasing quickly the contention window is not the answer to find the backoff function that fits better for improving network throughput. As Figure 4(b) shows there is a moment where $D C$ constant provide a worse throughput than $D C$ linear and $D C$ exponential. Figures
7 (a) and 7(b) show the percentage of the number of overlapping transmissions. The results concern the scenario with 32 active stations and is limited in two and three overlapping transmissions. These results emphasize the fact that collision probability do not depend on the packet size, since Figures 7 (a) and 7(b) are almost the same. It also show that the DC mechanism decreases collision, indeed. This is observed, because when the DC mechanism is not used, there is a lower percentage of collisions between two nodes and a higher percentage of collisions between three nodes. When the DC is not employed the overlapping transmissions have a higher probability and events like transmission overlappings involving three or more nodes are more frequent. Overlappings between a high number of stations is not desirable because it increases the number of 
retransmissions. We decide to evaluate the performance of a DC function, which provides te fastest growth for the contention window. This functions is a constant function with $k=0$. Figure 8 (a) shows that the $\mathrm{k} 0 \mathrm{DC}$ just performs better for a network with more than 16 active stations, for a packet size of 1,500 bytes. Additionally, for smaller packets KODC performance gets even worse, as shown in Figure 8(b). This result shows again that increasing fast does not mean a better performance. The best result depends on the packet size and the number of active stations.

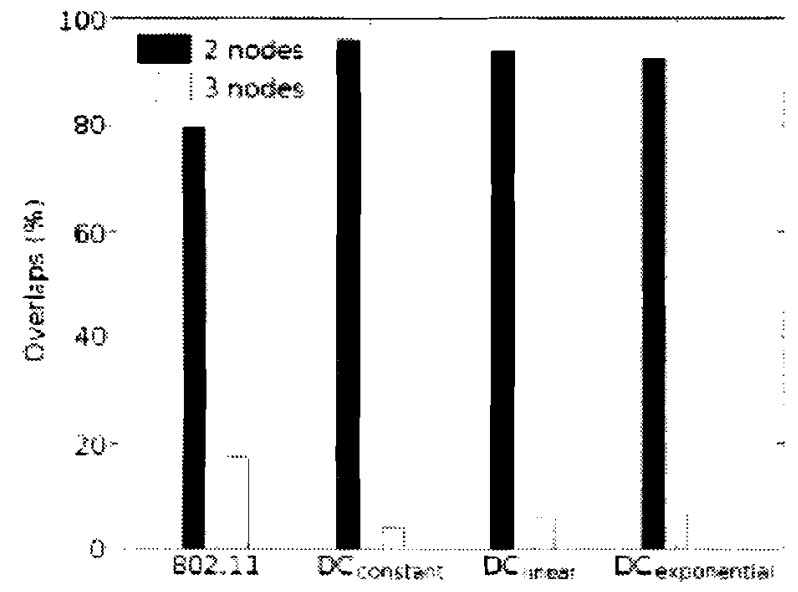

(a) Distribution for 1500 bytes packers

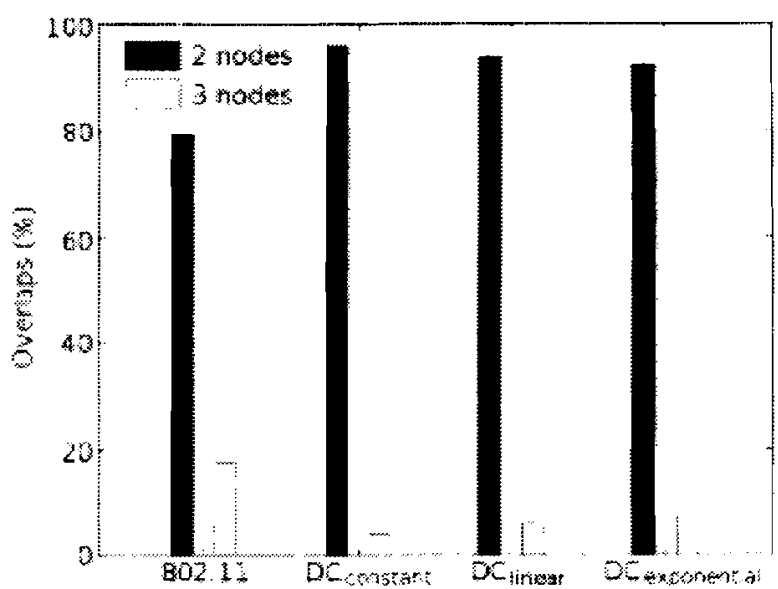

(b) Disangstion so 5l brte: packet:

Figure 7. Overlapping distribution with and without the analyzed mechanism.

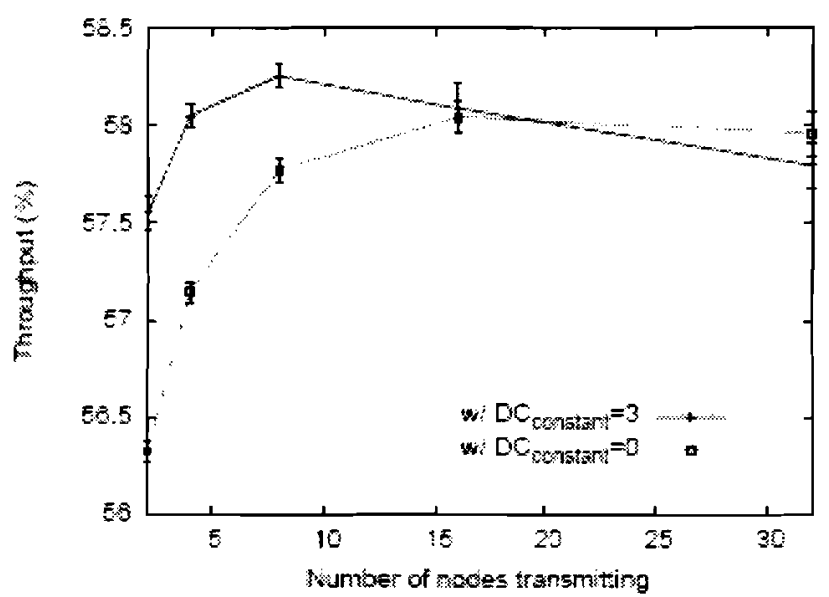

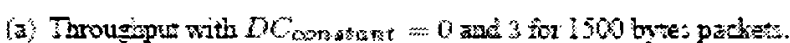

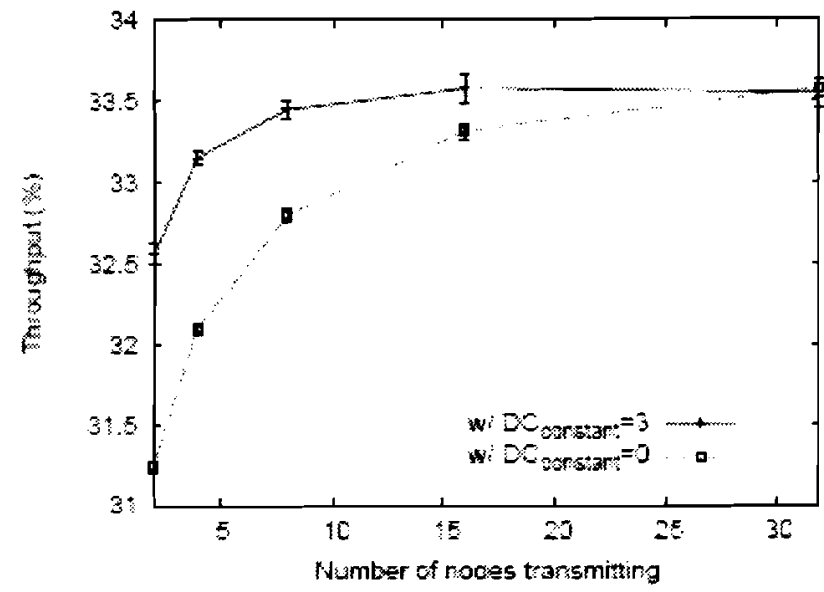

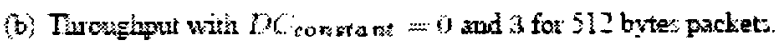

Figure 8. Throughput for different $D C$ constant values 


\section{CONCLUSIONS}

This paper presented and analyzed the performance of a collision avoidance mechanism to enhance the throughput and decrease the delay in 802.11 networks. The mechanismis based on the deferral counter (DC) algorithm proposed in HomePlug Standard. The purpose of the deferral counter is to increase the contention window faster than the regular backoff function from 802.11 to prevent collisions. Different from IEEE 802.11, the analysed mechanism increments the contention window of the deferring stations to minimize collisions. We implemented this mechanism in the ns-2 simulator in order to compare it with IEEE 802.11. We proposed the utilization of three different functions in the deferral counter mechanism and evaluated their performances considering network throughput, packet delay, and the percentage of collisions. Results showed that the proposed mechanism performs better when there is more than four active stations no matter the packet size. Most important, we showed that the backoff function which optimize better the network capacity depends on the packet size and the number of active nodes. This is mainly due to the tradeoff between the collision probability reduction achieved by the mechanism and the increased number of idle slots. Therefore, there is no simple DC function, that performs better than the others, for all situations. We plan, as future works, to propose a dynamic DC function, which adapts to the packet size and the number of active stations to optimize network throughput. A decreasing backoff function to minimize the idle slots and improve network capacity can also be studied.

\section{REFERENCES}

[Fall and Varadhan. 2002] Fall, K. and Varadhan. K. (2002). The ns Mamual. UC Berkeley, LBL, USC/ISI, and Xerox PARC. Available at http://www.isi.edu/nsnam/ns/ns-documentation.html. [IEEE, 1999] IEEE ( 1999). Wireless LAN medium access control (MAC) and physical layer (PHY) specifications. IEEE Standard 802.11

[IEEE, 2002] IEEE (2002). Part 3: Carrier sense multiple access with collision detection (CSMA/CD) access method and physical layer specifications. Revision of IEEE Std 802.3, 2000 Edition. [Kuo and Kuo, 2003] Kuo, W.-K. and Kuo, C.-C. (2003). Enhanced backoff scheme in CSMA/CA for IEEE 802.11. pages 2809-2813, Proc. IEEE VTC-Fall, Orlando, FL.

[Kwon et al., 2003] Kwon, Y., Fang, Y., and Latchman, H. (2003). A novel MAC protocol with fast collision resolution for wireless LANs. In Proc. IEEE Infocom 2003.

[L.Bononi et al., 1998] L.Bononi, M.Conti, and L.Donatiello (1998). Design and performance evaluation of a distributed contention control (dcc) mechanism for IEEE 802.11 wireless local area networks. pages 59-67, Proceedings of The First ACM International Workshop on Wireless Mobile Multimedia (WoWMoM'98), Dallas, Texas.

[Lee et al., ] Lee, M. K., Newman, R. E., Latchman, H. A., Katar, S., and Yonges, L.

[Li et al., 200I] Li, J., Blake, C., Couto, D. S. J. D., Lee, H. I., and Morris, R. (2001). Capacity of ad hoc wireless networks. In $A C M$ MOBICOM, pages 61-69.

[Tay and Chua, 2001] Tay, Y. C. and Chua, K. C. (2001). A capacity analysis for the IEEE 802.11 MAC protocol. Wireless Network, 7:159-171.
[Villela and Duarte, 2004] Villela, B. A. M. and Duarte, O. C. M. B. (2004). Maximum throughput analysis in ad hoc networks. In IFIP High Performance Networking - Networking 2004, Lectur Notes in Computer Science 3042, pages 223-234.

Pedro Braconnot Velloso was born in Rio de Janeiro, Brazil, on January 11, 1977. He received the Electronic Engineer degree and the M. Sc. degree in Electrical Engineering from Universidade Federal do Rio de Janeiro, Brazil. He is a D.Sc. student of Computer Science at LIP6 from Universit'e Pierre et Marie Currie. France. His major interests are in wireless communications, QoS guarantees, voice over IP, and home networks

Miguel Elias Mitre Campista was born in Rio de Janeiro, Brazil, on May 8, 1980. He received Telecommunication Engineer degree from Universidade Federal Fluminense, Brazil. He is a M.Sc. student of Electrical Engineering at GTA/COPPE-POLI from Universidade Federal do Rio de Janeiro, Brazil. His major interests are home networks, powerlines communications, and wireless communications.

Daniel de Oliveira Cunha was born in Rio de Janeiro, Brazil, on April 27, 1979. He received the Electronic Engineer degree and the M. Sc. degree in Electrical Engineering from Universidade Federal do Rio de Janeiro, Brazil. He is a D.Sc. student of Computer Science at Electrical Engineering at GTA/COPPE-POLI from Universidade Federal do Rio de Janeiro. Brazil. His major interests are wireless communications, sensor networks, energy conservation, and home networks.

Lu is Henrique Maciel Kosmalski Costa was born in Rio de Janeiro, Brazil, on October 4, 1973. He received the Electronic Engineer and M.Sc. in Electrical Engineering degrees from Federal University of Rio de Janeiro (UFRJ). Brazil, in 1997 and 1998, respectively. He defended his D.Sc. thesis at the University Pierre et Marie Curie (Paris 6), France, in 2001. Lu'is spent 2002 as a post-doc researcher at LIP6 (Laboratoire d'Informatique de Paris 6). Then, Luis was awarded a research grant from CAPES (agency of the Brazilian Ministry of Education), and moved to COPPE/UFRJ. Since August 2004, Lu'is is Associate Professor at the Federal University of Rio de Janeiro. His major research interests are in the area of routing, especially on wireless networks, group communication, quality of service, multicast, and stateless routing. Lu' 15 is member of IEEE and professional member of ACM.

Otto Carlos M. B. Duarte was born in Rio de Janeiro, Brazil, on October 23, 1953. He received the Electronic Engineer degree and the M. Sc. degree in Electrical Engineering from Universidade Federal do Rio de Janeiro, Brazil, in 1976 and 1981, respectively, and the Dr. Ing. degree from ENST/Paris, France, in 1985. Since 1978 he is Professor at Universidade Federal do Rio de Janeiro. From January 1992 to June 1993 he has worked at MASI Laboratory in Paris 6 University. In 1995, he has spent three months at International Computer Science Institute (ICSI) associated to the University of California at Berkeley. In 1999 and 2001, he worked as invited professor at Paris 6 University. He is heading the computer network group (Grupo de Teleinform'atica e Automac, ao - GTA). His major research interests are in multicast, QoS guarantees, security and mobile communications. 